\title{
Full-Duplex or Half-Duplex D2D Mode? Closed Form Expression of the Optimal Power Allocation
}

\author{
Hussein Chour ${ }^{1,2}$, Youssef Nasser ${ }^{3}$, Oussama Bazzi ${ }^{2}$, and Faouzi Bader ${ }^{1}$ \\ ${ }^{1}$ CentraleSupélec/IETR, CentraleSupélec Campus de Rennes, 35510 Cesson-Sévigné, France \\ ${ }^{2}$ Physics and Electronics Department, Faculty of Science 1, Lebanese University, Beirut, Lebanon \\ ${ }^{3}$ ECE Department, American University of Beirut, Bliss Street, Beirut, Lebanon, \\ Email:\{hussein.chour@supelec.fr, yn10@aub.edu.lb, obazzi@ul.edu.lb, faouzi.bader@supelec.fr\}
}

\begin{abstract}
In this paper, we investigate and derive a closedform expression for the power allocation scheme of full duplex (FD) device to device (D2D) communications underlaying wireless cellular network. In this scenario, we consider the FD-D2D pair sharing the uplink resources of cellular users. We first derive a closed-form expression for the ergodic rate of the D2D link. Then we formulate the optimization problem which aims to maximize the D2D link rate while fulfilling the minimum QoS requirement of the cellular user. We further derive a closedform expression for the optimal power allocation strategy for both D2D and cellular users. The simulation results show the accuracy of the derived power allocation scheme and provide important insights on the separation distance between the D2D users and the interfering cellular user. In addition, the results provide important conditions to switch between FD and half duplex (HD) D2D modes.
\end{abstract}

Index Terms-full-duplex transmission, device-to-device (D2D) communication, optimal power allocation, full-duplex versus halfduplex

\section{INTRODUCTION}

With the rapidly growing of the customers data traffic demand, improving the system capacity and increasing the user throughput have become essential concerns for the future wireless communication network, i.e. 5G. In this context, D2D communication and FD are proposed as a potential solutions to increase the spatial spectrum utilization and the user rate in a cellular network [1]. D2D allows two nearby devices to communicate without base station participation or with a limited participation. On the other hand, FD communication allows simultaneous transmission and reception in the same frequency band. Hence, it will enhance the spectral efficiency of a single peer-to-peer channel and will improve the users throughput (potentially doubled) over the conventional halfduplex (HD) communication [2].

The main challenge in FD communication is the strong selfinterference (SI) imposed on the receiver by the node's own transmitter [3]. However, the recent works on FD show that huge advances have been made in mitigating the SI, and the respective state of the art of transceiver design can achieve a high level of self-interference cancellation (SIC) [4] [5]. Hence, the FD technology is getting closer to being applied in the new wireless cellular network.

The short distance property of the D2D link makes the transmission power of the D2D users relatively lower. Thus, exploiting the FD transmission in D2D communication is an excellent choice to further improve the cellular spectrum efficiency and the users throughput [6]. However, the full duplex D2D (FD-D2D) communication adds new challenges for the D2D communication. For example, the amount of SI is highly depending on the transmitted power value. Thus, the power allocation strategy in FD-D2D is a very important problem to be tackled.

The authors of [7] derived and analyzed a closed form expression of the sum-rate of a FD-D2D enabled cellular network. However, in [7] the optimal power allocation scheme was not considered. Moreover, only a symmetric scenario, in which the D2D users have the same distance to the base station from one side and the cellular user (CUE) has equal distance to the D2D users from the other side, was assumed therein. The power optimization problem of an isolated FD-D2D pair underlay cellular network was tackled in [8]. In [9], the authors derived a convex optimization problem to maximize the rate of FD-D2D link while satisfying the minimum rate requirement of the cellular users. Although the solution derived in [9] is not limited to the case where the distances between the D2D users and the CUE are equal, the authors of [9] neglected the effect of the CUE location w.r.t the D2D users and they did not clearly described the D2D pair situation in their numerical results. Moreover, neither [8] nor [9] provided a mathematical expression for the optimal power allocation scheme.

In this paper a mathematical framework of the FD-D2D communication is developed, and advanced analysis of the impact of the CUE location on the optimal FD-D2D rate is provided. Precisely, the derived framework covers both the symmetric and the asymmetric scenarios of the D2D users. Throughout the paper, the symmetric case means that the D2D users are receiving the same interference power from the CUE and they are introducing equal interference power at the BS, i.e., the CUE has equal distance to the D2D users and the D2D users have equal distance to the base station. The asymmetric case reflects the situation where one of the D2D users introduces more interference on the BS than the other, or one of the D2D users is receiving more interference from the CUE than the other or both. The key contributions of this work are as follows: 
- Derivation of the closed-form expressions for the average FD-D2D rate, HD-D2D rate and for the optimal power allocation strategy. To the best of the authors knowledge, there is no existing work that provides such expressions considering both the symmetric and the asymmetric cases.

- Derivation of the mathematical expressions which characterize the requirements to have a reliable FD-D2D versus HD-D2D modes.

- Shedding the light on the impact of the CUE location on the FD-D2D rate.

The rest of this paper is organized as follows. Section II describes the system model and section III derives the FDD2D ergodic capacity. The optimal power allocation strategy is presented in section IV. In section V we discuss the simulation results, and the conclusion is drawn in section VI.

\section{SYSTEM MODEL}

As depicted in Fig. 1, we consider a FD-D2D enabled cellular network. The cellular network consists of an eNodeB, one D2D pair and multiple CUEs. The CUEs are allocated orthogonal sub-channels for uplink transmission and they are assumed to operate in HD mode while the FD-D2D link can share only one uplink subchannel so there is interference only between one cellular user $\left(C U E_{i}\right.$ in Fig. 1$)$ and the D2D pair.

Although the advanced transceiver designs can significantly decrease the SI, in practical scenario it is impossible to totally delete it. In this work, the power of the residual selfinterference $\left(P_{R S I}\right)$ is defined as follows:

$$
P_{R S I}=\eta P_{t}
$$

where $\eta(0 \leq \eta \leq 1)$ is the SI mitigation coefficient which represents the effect of the advanced SIC techniques [8] [9], and $P_{t}$ is the local transmit power. The case of $\eta=0$ corresponds to the perfect SIC while $\eta=1$ reflects the invalidity of SIC.

To model the cellular/FD-D2D links as well as the interference links, both the distance based path-loss model and the fast fading are considered. Thus the channel gain between a transmitter $i$ and a receiver $j$ can be expressed as $g_{i j}=l_{i j} \cdot h_{i j}$, where $l_{i j}$ denotes the path-loss attenuation and $h_{i j}$ stands for the Rayleigh distributed fading coefficient with unit mean power gain (i.e., $h_{i j} \sim \exp (1)$ ). Furthermore, the path-loss attenuation can be expressed by $l_{i, j}=d_{i j}^{-\alpha}$ where $\alpha$ is the standard path loss exponent and $d_{i j}$ is the distance between the transmitter $i$ and the receiver $j$. Considering an interference limited system as in [10], [11], i.e., the receiver noise is negligible, the instantaneous Signal-to-Interference ratios (SIRs) of the CUE, of the first D2D user (D1) and of the second D2D user (D2) can be respectively expressed as:

$$
\begin{gathered}
\gamma_{c}=\frac{P_{c} l_{c, B S} h_{c, B S}}{P_{d 1} l_{d 1, B S} h_{d 1, B S}+P_{d 2} l_{d 2, B S} h_{d 2, B S}} \\
\gamma_{d 1}=\frac{P_{d 2} l_{d} h_{d 2, d 1}}{P_{c} l_{c, d 1} h_{c, d 1}+\eta P_{d 1}} \\
\gamma_{d 2}=\frac{P_{d 1} l_{d} h_{d 1, d 2}}{P_{c} l_{c, d 2} h_{c, d 2}+\eta P_{d 2}}
\end{gathered}
$$

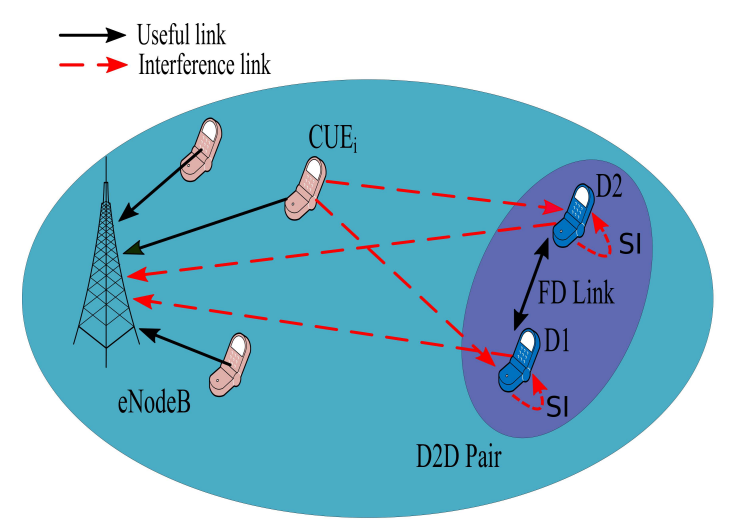

Fig. 1. A FD-D2D pair shares the resources of one cellular user, which creates interference between the two types of links.

where $P_{c}, P_{d 1}$ and $P_{d 2}$ are the transmit powers of the $C U E, D 1$, and $D 2$ respectively. $l_{d}$ is the path-loss attenuation between the D2D users.

\section{ERGODIC CAPACITY ANALYSIS}

In this section, we provide the analytical derivations and analysis of the ergodic capacity expressions for the different devices in the network, i.e. CUE, D1 and D2. For the sake of fairness, the analysis is provided for the two modes: FD and HD.

\section{A. The Full Duplex Mode}

In this subsection, we will first provide a closed form expression for the ergodic capacity of a FD-D2D link $\left(R_{F D}\right)$ which is using the CUE uplink resources. $R_{F D}$ is defined as the summation of D1's and D2's ergodic capacities, $R_{D 1}$ and $R_{D 2}$ in (5), and it is given by:

$$
R_{F D}=\underbrace{\mathbb{E}_{\gamma_{d 1}}\left[\log _{2}\left(1+\gamma_{d 1}\right)\right]}_{R_{D 1}}+\underbrace{\mathbb{E}_{\gamma_{d 2}}\left[\log _{2}\left(1+\gamma_{d 2}\right)\right]}_{R_{D 2}}
$$

where $\gamma_{d 1}$ and $\gamma_{d 2}$ are the SIRs given by (3) and (4) respectively. $\mathbb{E}($.$) denotes the expectation operation. From (5), in$ order to derive the FD ergodic capacity, the probability density function (PDF) of the SIRs must be calculated. The following Lemmas provide such PDFs.

Lemma 1. The distribution of the CUE SIR is given by:

$$
\begin{aligned}
f_{\gamma_{c}}(t)= & \left(\frac{P_{c} l_{c, B S}}{P_{d 1} l_{d 1, B S}-P_{d 2} l_{d 2, B S}}\right) \\
& {\left[\frac{\left(P_{d 1} l_{d 1, B S}\right)^{2}}{\left(t P_{d 1} l_{d 1, B S}+P_{c} l_{c, B S}\right)^{2}}\right.} \\
& \left.-\frac{\left(P_{d 2} l_{d 2, B S}\right)^{2}}{\left(t P_{d 2} l_{d 2, B S}+P_{c} l_{c, B S}\right)^{2}}\right]
\end{aligned}
$$

Proof: First let us define the two following random variables: $X=P_{c} l_{c, B S} h_{c, B S}$ and $Y=P_{d 1} l_{d 1, B S} h_{d 1, B S}+$ $P_{d 2} l_{d 2, B S} h_{d 2, B S}$. The channel power gains follow the exponential distribution. Therefore, the PDF of $X$ and $Y$ are $\frac{1}{P_{c} l_{c, B S}} e^{-\frac{x}{P_{c} l_{c, B S}}}$ and $\frac{e^{-\frac{y}{P_{d 1} l_{d 1, B S}}}-e^{-\frac{y}{P_{d 2} l_{d 2, B S}}}}{P_{d 1} l_{d 1, B S}-P_{d 2} l_{d 2, B S}}$ respectively. 
By defining new variables $S=Y$ and $T=X / Y$ and applying the change of variable theorem, the joint PDF for the couple $(S, T)$ can be written as:

$$
\begin{aligned}
f_{S T}(s, t) & =f_{X Y}(X(s, t), y(s, t))|J(s, t)| \\
& =\frac{s e^{\frac{s t}{P_{c} l} c, B S}}{P_{c} l_{c, B S}}\left[\frac{e^{-\frac{s}{P_{d 1} l d, B S}}-e^{-\frac{s}{P_{d 2} l d, B S}}}{P_{d 1} l_{d 1, B S}-P_{d 2} l_{d 2, B S}}\right]
\end{aligned}
$$

where, $|J(s, t)|$ is the Jacobian of the transformation. Now, integrating (7) w.r.t to $s$ completes the proof.

Lemma 2. The PDF of the D1 and D2 SIRs are given by:

$$
\begin{aligned}
f_{\gamma_{d 1}}(t)= & e^{t \frac{\eta P_{d 1}}{P_{d 2} l_{d}}} \\
& \frac{\left(P_{d 1} P_{c} l_{d} l_{c, d 1}+\eta P_{d 1} P_{d 2} l_{d}+t \eta P_{c} P_{d 1} l_{c, d 1}\right)}{\left(t P_{c} l_{c, d 1}+P_{d 1} l_{d}\right)^{2}} \\
f_{\gamma_{d 2}}(t)= & e^{t \frac{\eta P_{d 2}}{P_{d 1} l_{d}}} \\
& \frac{\left(P_{d 2} P_{c} l_{d} l_{c, d 2}+\eta P_{d 1} P_{d 2} l_{d}+t \eta P_{c} P_{d 2} l_{c, d 2}\right)}{\left(t P_{c} l_{c, d 2}+P_{d 1} l_{d}\right)^{2}}
\end{aligned}
$$

Proof: The PDF of $\gamma_{d 1}$ and $\gamma_{d 2}$ can be obtained by following the same procedure as in Lemma 1.

Now, putting the D2D SIR distributions derived in Lemma 2 in (5) and integrating the Shannon capacities over the SIRs leads to the following theorem.

Theorem 1. The full duplex ergodic capacity is given by:

$$
\begin{aligned}
R_{F D}= & \underbrace{\frac{l_{c, d 1}}{l_{d} y}-1}_{R_{D 1}\left(\frac{\eta x}{l_{c, d 1}}\right) e^{\frac{\eta x}{l_{c, d 1}}}-E_{1}\left(\frac{\eta x}{l_{d} y}\right) e^{\frac{\eta x}{l_{d} y}}} \\
+ & \underbrace{\frac{E_{1}\left(\frac{\eta y}{l_{c, d 2}}\right) e^{\frac{\eta y}{l_{c, d 2}}}-E_{1}\left(\frac{\eta y}{l_{d} x}\right) e^{\frac{\eta y}{l_{d} x}}}{\frac{l_{c, d 2}}{l_{d} x}-1}}_{R_{D 2}}
\end{aligned}
$$

where $x=\frac{P_{d 1}}{P_{c}}, y=\frac{P_{d 2}}{P_{c}}$ and $E_{1}(z)=\int_{z}^{\infty} \frac{e^{-t}}{t} d t$ is the first order exponential integral.

Note that the function

$$
h(x) \triangleq \exp \left(\frac{1}{x}\right) E_{1}\left(\frac{1}{x}\right)
$$

is a monotonically increasing function with $x$ [12]. Accordingly, for $a>b$ ( $a$ and $b$ are arbitrarily positive numbers)

$$
\exp \left(\frac{\eta y}{a}\right) E_{1}\left(\frac{\eta y}{a}\right)>\exp \left(\frac{\eta y}{b}\right) E_{1}\left(\frac{\eta y}{b}\right)
$$

then we have

$$
\frac{b}{a-b}\left[e^{\frac{\eta y}{a}} E_{1}\left(\frac{\eta y}{a}\right)-e^{\frac{\eta y}{b}} E_{1}\left(\frac{\eta y}{b}\right)\right]>0
$$

The same result can be obtained for $a<b$. Thus, $R_{D 1}$ and $R_{D 2}$ are always positive and monotonically increasing with respect to $y$ and $x$ respectively. This in turn verifies that the FD capacity derived in (10) is always positive.

\section{B. The Half Duplex Mode}

In FD communication the D2D users share the whole CUE's spectrum while in HD only one D2D user can use the CUE's resources. Thus, for fair comparison $R_{H D}$ is assumed to be the maximum of the HD rate of $D 1$ and $D 2$ as presented in (14).

$$
R_{H D}=\max (\underbrace{\frac{\log _{2}\left(\frac{l_{c, d 1}}{l_{d} y}\right)}{\frac{l_{c, d 1}}{l_{d} y}-1}}_{R_{D 1}^{H D}}, \underbrace{\frac{\log _{2}\left(\frac{l_{c, d}}{l_{d} x}\right)}{l_{c, d 2}}-1}_{R_{H D}^{D 2}})
$$

where $R_{D 1}^{H D}$ and $R_{D 2}^{H D}$ are the HD of $D 1$ and $D 2$ and they can be easily calculated by following the same procedure as in Lemma.1. It is worth mentioning that from the derivations perspectives, the HD mode could be seen as a sub-case of the FD mode. Hence, we will focus in the next section on the FD mode only.

\section{MaXimizing the Full Duplex D2D ERGodiC CAPACITY}

The aim of this section is to maximize the FD-D2D capacity while satisfying the QoS requirement of the interferer CUE by finding the optimal power allocation scheme. Thus, the maximization problem denoted by $\boldsymbol{P} \mathbf{1}$ can be formulated as,

$$
\begin{aligned}
& \text { P1: } \max _{\mathbf{P}} R_{F D}(\mathbf{P})=R_{D 1}+R_{D 2} \\
& \text { s.t. } \mathbb{E}\left[I_{B S}\right] \leq I^{\max }
\end{aligned}
$$

where, $\mathbf{P}=\left[x=\frac{P_{d 1}}{P_{c}}, y=\frac{P_{d 2}}{P_{c}}\right]$ is the power ratio variable vector, $\mathbb{E}\left[I_{B S}\right]$ is the average interference power at eNodeB, and $I^{\max }$ denotes the maximum interference power that is acceptable at the BS. The utility function in (15) is the FDD2D capacity presented in (10), while the constraint in (15a) keeps the interference of the D2D users at a certain level and thus it reflects the QoS of the CUE. To obtain the optimal power allocation scheme, it is highly desirable that $\boldsymbol{P 1}$ is a concave optimization problem. The next subsection will analyze the concavity of $\boldsymbol{P} \mathbf{1}$.

\section{A. Analysis of the full duplex rate}

From (10), $R_{F D}$ is defined if $y \neq \frac{l_{c, d 1}}{l_{d}}$ and $x \neq \frac{l_{c, d 2}}{l_{d}}$. Accordingly, the FD capacity is defined over four regions $\mathcal{R}_{1}=\left\{y<\frac{l_{c, d 1}}{l_{d}}, x>\frac{l_{c, d 2}}{l_{d}}\right\}, \mathcal{R}_{2}=\left\{y>\frac{l_{c, d 1}}{l_{d}}, x<\frac{l_{c, d 2}}{l_{d}}\right\}$, $\mathcal{R}_{3}=\left\{y<\frac{l_{c, d 1}}{l_{d}}, x<\frac{l_{c, d 2}}{l_{d}}\right\}$ and $\mathcal{R}_{4}=\left\{y>\frac{l_{c, d 1}}{l_{d}}, x>\frac{l_{c, d 2}}{l_{d}}\right\}$. However, the main FD gain can only be achievable in $\mathcal{R}_{4}$ as shown in Appendix A. Otherwise, the FD gain will be less than $1 b i t / s / H z$. Intuitively, the FD gain is considerable in $\mathcal{R}_{4}$ where the average power of the useful signal is greater than the average interference power. The problem shrinks down to study the utility function in this region. Using the derivations in Appendix B, it can be shown that the utility function (15) is concave in $\mathcal{R}_{4}$.

On the other hand, to guarantee the QoS constraint on the CUE we need to ensure that the average interference power at the eNodeB is less than a predetermined threshold $I^{\text {max }}$. Based on Conjuncture 1, defined in Appendix A, the average 
power of the useful signal received by the BS (i.e. the CUE signal) has been chosen as $I^{\max }$.

$$
\underbrace{\sum_{i=1}^{2} P_{d i} l_{d i, B S}}_{\mathbb{E}\left[I_{B S}\right]} \leq \underbrace{P_{c} l_{c, B S}}_{I^{\max }}
$$

Moreover, it is straight forward to see that the maximum FD capacity rate occurs when the interference power of the D2D is at the maximum level (i.e. the equality case in (16)). Now, solving (16) with equality leads to the following relation between the powers:

$$
l_{c, B S}=l_{d 1, B S} x+l_{d 2, B S} y
$$

where $x$ and $y$ are the power ratios defined in Theorem 1 .

As it can be seen, (17) is linear. Thus, the problem $\boldsymbol{P 1}$ is concave.

\section{B. The Optimal power allocation scheme}

Here, we tackle the problem of power allocation which requires solving (15) taking into account (15a). However, this problem appears to be mathematically intractable as the optima can not be directly obtained.

To propose a suitable solution, we split our problem to two sub-cases named symmetric and asymmetric scenarios. The symmetric scenario represents the case where the D2D users have equal distance from the BS (i.e, $l_{d 1, B S}=l_{d 2, B S}$ ) and the CUE has equal distance from the D2D users (i.e, $l_{c, d 1}=l_{c, d 2}$ ) (refer to Fig. 2), while the asymmetric scenario covers all the remaining possible users locations (see Fig. 3).

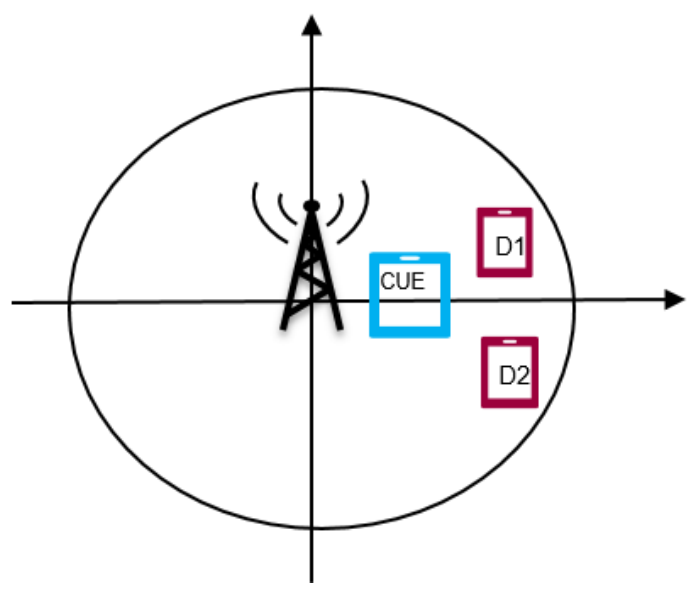

Fig. 2. Symmetric scenario $\left(d_{d 1, B S}=d_{d 2, B S} \& d_{c, d 1}=d_{c, d 2}\right)$

Power Allocation in the Symmetric Case: Let us consider that the interference power is much lower than the average received power, i.e., $y>>l_{c, d 1} / l_{d}$ and $x>>l_{c, d 2} / l_{d}$. Under this condition, we found that the optima of the symmetric case can be easily calculated by applying (17) into (15) and solving its derivative. The optima in such case is,

$$
x_{\text {sym }}^{*}=y_{\text {sym }}^{*}=\frac{M_{x}}{2}=\frac{l_{c, b s}}{2 l_{d 1, b s}}
$$

where $M_{x}$ is the maximum allowed power ratio for $D 1$. Physically speaking, (18) means that when the D2D users are receiving the same interference power from CUE and introducing equal interference power at the BS, the optima can be obtained by allocating half the maximum power for each D2D user. Moreover, from Appendix A we know that (18) is true only when $\eta<l_{d}$ otherwise the D2D pair must switch to the HD mode.

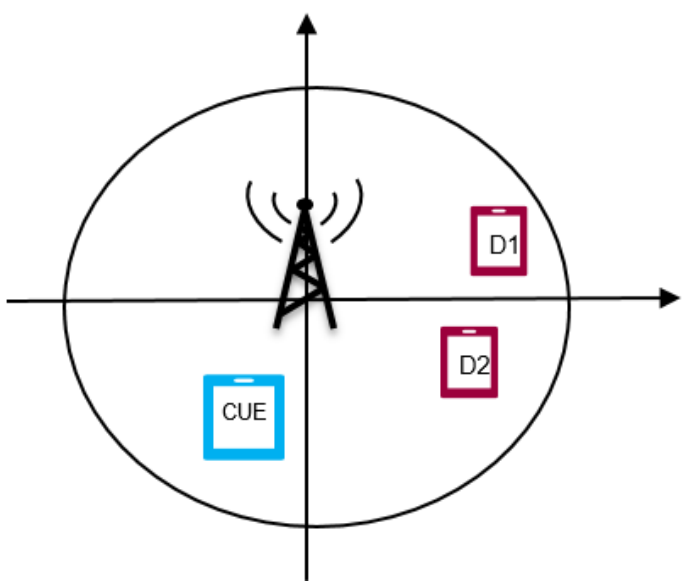

Fig. 3. Asymmetric scenario $\left(d_{d 1, B S} \neq d_{d 2, B S}\right.$ or $\left.d_{c, d 1} \neq d_{c, d 2}\right)$

Power Allocation in the Asymmetric Case: For the asymmetric case, the optima is given by:

$$
x_{\text {asym }}^{*}=\frac{A-\sqrt{B}}{2 \eta \beta\left(\beta l_{c, d 1}-l_{c, d 2}\right)} \text { and } y_{\text {asym }}^{*}=M_{y}-\beta x_{\text {asym }}^{*}
$$

where

$$
M_{y}=\frac{l_{c, B S}}{l_{d 2, B S}}
$$

is the maximum allowed power ratio for $D 2$, $\beta=l_{d 1, B s} / l_{d 2, B s}, A=4 \eta \beta l_{c, d 1} M+3 \beta l_{c, d 1} l_{c, d 2}$, and $B=A^{2}-\left[\left(l_{c, d 1}-l_{c, d 2}\right)\left(16 M^{2} \eta^{2} \beta l_{c, d 1}+24 M \beta \eta l_{c, d 1} l_{c, d 2}\right)\right]$.

Proof: Using the properties of $E_{1}(z)$ given in [13], the function $E_{1}(z) \exp (z)$ can be tightly approximated by $\ln (1+$ $\left.\frac{1}{z}\right)$. Moreover, assuming that the average received power is much larger than the interference power, $R_{F D}$ can be written as follows:

$$
R_{F D} \approx \ln \left(1+\frac{l_{d} y}{\eta x}\right)+\ln \left(1+\frac{l_{d} x}{\eta y}\right)-\ln \left(1+\frac{l_{c, d 1}}{\eta x}\right)-\ln \left(1+\frac{l_{c, d 2}}{\eta y}\right)
$$

Now applying (17) into (21) and solving the derivative of the result leads to the optimal power ratios in (19)

The obtained optima in (18) and (19) are for two particular cases. For the general case where the SI factor can be any number and the users are in random positions the optima can not be mathematically derived. However, after analyzing (19) we found that when CUE is relatively far from the D2D pair the optima will be achieved by allocating more power to the D2D user who is introducing less interference power to the base station. And, when the CUE is relatively near to the $\mathrm{D} 2 \mathrm{D}$ pair, more power should be allocated to the D2D 
user who is suffering more from the CUE interference power. Based on this analysis and the previous results we propose the following power allocation technique which yields to the optimum results in both the symmetric and asymmetric case.

$$
\begin{aligned}
x^{*} & =\max \left(0, \min \left(M_{x}, e^{\frac{\eta M}{l_{d}}} \frac{M_{x}}{2}\left[1+\frac{l_{c, d 1}-l_{c, d 2}}{l_{d}+l_{c, d 1}+l_{c, d 2}}\right]\right)\right) \\
y^{*} & =\frac{l_{c, b s}-l_{d 1 . b s} x^{*}}{l_{d 2, b s}}
\end{aligned}
$$

The $\max ()$ and $\min ()$ operators in $(22)$ are used to ensure that our approximated optima do not violate the power ratio constraints, i.e., the minimum power ratio 0 and the maximum power ratio $M_{x}$. The exponential term reflects the effect of the SIC techniques, more precisely when $\eta$ goes to zero the effect of SI will disappear while when $\eta$ goes to one the SI will highly affect the optima solution. The fraction term in (22) will be positive or negative w.r.t the CUE location and thus more power will be allocated to D1 if it is suffering more from the CUE interference and vice versa. Accordingly, depending on the CUE location the value of the optima will tend to either $\left(M_{x}, 0\right)$ or $\left(0, M_{y}\right)$ which represent the HD-D2D mode for $D 1$ and $D 2$ respectively. In other word, the CUE location highly affects the transmission mode for the D2D pair. The accuracy of this approximation will be validated in the numerical simulation section when comparing the derived optimal solution with the exhaustive search results.

\section{NUMERICAL RESULTS}

In this section, we conduct numerical experiments to evaluate the performance of our proposed optimal power allocation. For our simulation we assume a circular cell of radius $300 \mathrm{~m}$. The maximum distance between the D2D users is assumed to be $40 \mathrm{~m}$ as in [8][9]. Moreover, we set the path-loss exponent, $\alpha$, to 3 and we varied the value of the SI mitigation factor $\eta$ between two realistic values $-60 \mathrm{~dB}$ and $-90 \mathrm{~dB}$ [4]-[9].

Fig. 4 and Fig. 5 validate the optimal power allocation for the symmetric and asymmetric cases respectively. For the symmetric case we set the D2D pair at a distance $100 \mathrm{~m}$ from the eNodeB then we moved the CUE on the median line which intersects with the line $\overline{D 1 D 2}$ in the triangle formed by the eNodeB, $D 1$ and $D 2$ (refer to Fig.2). In the asymmetric case, first we fixed $D 1$ at a distance $100 \mathrm{~m}$ from the base station then we set $D 2$ at a random position such that $d_{d 2, b s}>d_{d 1, b s}$ and $d_{d} \leq 40 \mathrm{~m}$. After that, we set the CUE at a random position such that $d_{c, b s}<d_{d 1, b s}$ and finally we moved CUE toward $D 1$. This scenario allows us to study the cases in which the CUE is close or far from $D_{1}$ which in turn allows us to validate the power allocation strategy in the asymmetric scenario. As it can be seen, the results obtained from our power allocation are very close to the exhaustive search results. Hence, the derived equations are valid and thus they can be used to maximize the rate. In addition, from both Fig. 4 and Fig. 5 we can see that as $\eta$ decreases the FD rate increases. The reason behind that is when $\eta$ decreases the power of the residual self interference decreases and hence the average interference power decreases. Fig. 4 also shows that as the distance between the CUE and

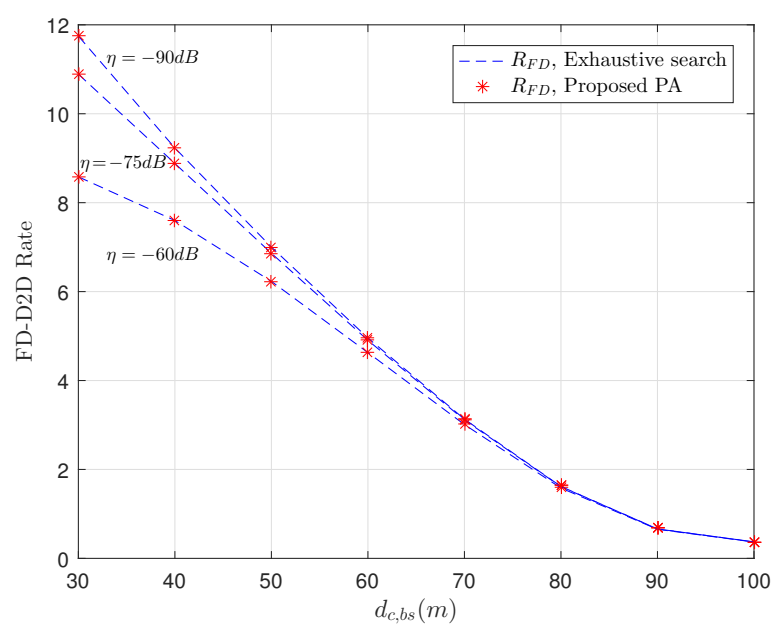

Fig. 4. Comparison of FD-D2D rate obtained from the exhaustive search and from the proposed power allocation scheme in the symmetric case

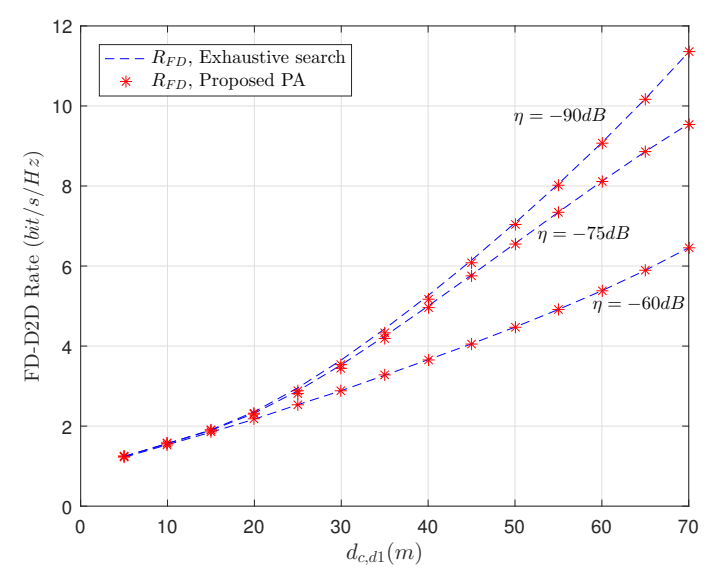

Fig. 5. Comparison of FD-D2D rate obtained from the exhaustive search and from our proposed power allocation scheme in the asymmetric case

the base station increases the FD-D2D rate decreases. This is expected behavior because in such case the D2D users need to mitigate their transmission power to not violate the interference constraint at the base station. Moreover, Fig. 5 shows that when the CUE is very close to one of the D2D user (e.g., $d_{c, d 1}=5 \mathrm{~m}$ ), the maximum D2D capacity occurs by allocating the whole allowed power to that user. This is why the depicted results in Fig. 5 converge to the same value as the interferer becomes closer to $D 1$.

Now in order to show the high effect of the CUE location on the FD-D2D rate, we first fix the D2D pair at $100 \mathrm{~m}$ from the BS, then we set the CUE at a position where $d_{c, d 1}>d_{c, d 2}$. After that we moved the CUE toward $D 1$ or $D 2$. We repeated this scenario for different values of $l_{d}$. Fig. 6 shows the variations of the optimal power ratios with respect to the cellular user distances from the D2D users in such situation. As expected, $x^{*}$ and $y^{*}$ have opposite variation w.r.t the CUE location. For instance, when $d_{c, d 1}=d_{c, d 2}$ we have $x^{*}=y^{*}=50$. While when the CUE becomes too close 


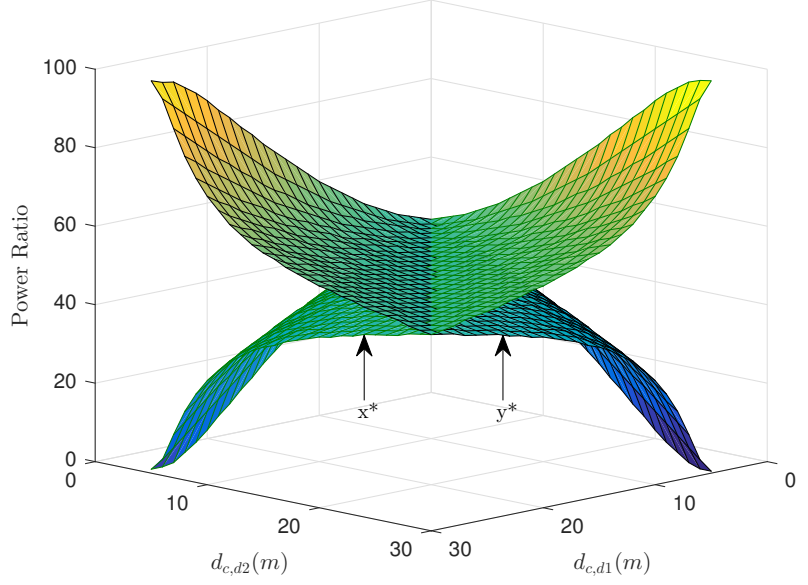

Fig. 6. The optimal power ratios variation w.r.t to CUE location. $(\eta=-70 d B)$

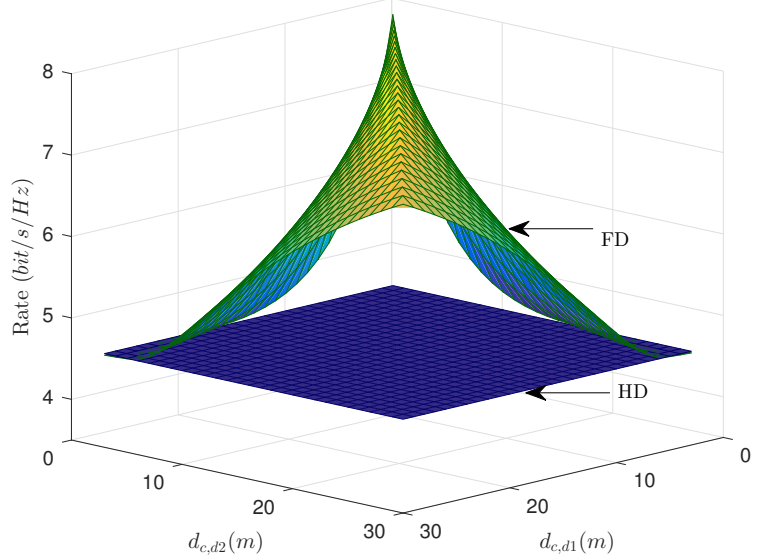

Fig. 7. The effect of the CUE location on the FD rate. $(\eta=-70 d B)$ to $D 1$ (i.e., $\left.d_{c, d 1}=5 m\right), x^{*}$ is almost equal to the maximum allowed power ratio and $y^{*}$ is almost zero. This is because in such case $D 1$ is facing high interference from the CUE while $D 2$ is not. Hence, it is better to let $D 1$ only sending messages, i.e., it will operate in HD mode. Fig. 7 shows the optimal rate variation with respect to $l_{c, d 1}$ and $l_{c, d 2}$. In addition, it compares the optimal FD rate with the optimal HD rate. As can be seen, the maximum FD gain occurs when $d_{c, d 1}=d_{c, d 2}$ while in the asymmetric case the FD rate decreases with the decreasing of $d_{c, d 1}$ or $d_{c, d 2}$. Hence, when the CUE is too close to one of the D2D users the FD has no rate gain. Finally, Fig. 7 clearly shows that the main gain of full duplex occurs when the CUE is far enough from the D2D pair.

\section{CONCLUSION}

In this paper, we investigated the power allocation problem of a FD-D2D based cellular network. In particular, we formulated an optimization problem to maximize the FDD2D rate while fulfilling the minimum QoS requirement of CUE. We further derived a closed-form expression for the optimal power allocation strategy. On contrary to the related works, the derived solution covered both the symmetric and the asymmetric scenarios. The simulation results proved the derived equation and showed that the distance from the interferer cellular user, the distance between the D2D pair, and the SIC factor have a great impact on the FD-D2D ergodic capacity and the power allocation scheme. For instance, when CUE is relatively far from the D2D pair the optima will be achieved by allocating more power to the D2D user who is introducing less interference power to the base station. While when the CUE is relatively near the D2D pair, more power should be allocated to the D2D user who is suffering more from the CUE interference power. Finally, both simulation and analysis showed that the maximum FD-D2D rate occurs when the D2D users are sharing the CUE' spectrum who is located near the BS and far away from the D2D pair. As a result the users location or in general the users' interference highly affects the D2D rate and thus it should be well treated in the resource allocation phase.

\section{APPENDIX A}

We begin our proof by providing the following conjecture.

Conjecture 1. In wireless communication the receiver decodes correctly the signals when the following inequality holds:

$$
\mathbb{E}[P] \geq \mathbb{E}[I]
$$

where $\mathbb{E}[P]$ is the average power of the useful signal and $\mathbb{E}[I]$ is the average interference power.

Now by applying Conjecture 1 on (3) and (4) we can see that the FD communication can be useful only when the SIC factor, $\eta$, is less than a certain value as follows:

$$
\eta<\min \left(\frac{y l_{d}-l_{c, d 1}}{x}, \frac{x l_{d}-l_{c, d 2}}{y}\right)
$$

Since $\eta$ is positive, the following inequalities must be satisfied:

$$
x>\frac{l_{c, d 2}}{l_{d}} \text { and } y>\frac{l_{c, d 1}}{l_{d}}
$$

Note that, (24) is the same as $\mathcal{R}_{4}$. Moreover, if (24) does not hold the SIC factor will be negative which means that the FD transmission is unsuitable in such situation. Thus, the D2D users must switch to the HD mode. Now in order to investigate the rates in the remaining regions we derived the limits of $R_{D 1}, R_{D 2}, R_{D 1}^{H D}$, and $R_{D 2}^{H D}$, which are respectively defined in (10) and (14), as follows:

$$
\begin{aligned}
& \lim _{y \rightarrow \frac{l_{c, d 1}}{l_{d}}} R_{D 1}=1-\frac{\eta x}{l_{c, d 1}} E_{1}\left(\frac{\eta x}{l_{c, d 1}}\right) e^{\frac{\eta x}{l_{c, d 1}}} \\
& \lim _{x \rightarrow \frac{l_{c, d 2}}{l_{d}}} R_{D 2}=1-\frac{\eta y}{l_{c, d 2}} E_{1}\left(\frac{\eta y}{l_{c, d 2}}\right) e^{\frac{\eta y}{l_{c, d 2}}} \\
& \lim _{y \rightarrow \frac{l_{c, d 1}}{l_{d}}} R_{D 1}^{H D}=1, \quad \lim _{x \rightarrow \frac{l_{c, d 2}}{l_{d}}} R_{D 2}^{H D}=1
\end{aligned}
$$

Based on (25) and the monotonic increasing property of the D2D users rate, the maximum rate of $D 1$ and $D 2$ when $y<\frac{l_{c, d 1}}{l_{d}}$ and $x<\frac{l_{c, d 2}}{l_{d}}$ will be $1 b i t / s / H z$ respectively. Hence, the FD transmission can show its efficiency only in $\mathcal{R}_{4}$. 
Using Conjecture.1, (3), and (4) the minimum distance between the CUE and the D2D devices to have reliable FD communication link can also be derived as follows:

$$
\begin{aligned}
& l_{c, d 1}<y l_{d}-\eta x \Leftrightarrow d_{c, d 1}>\left(y l_{d}-\eta x\right)^{-1 / \alpha} \\
& l_{c, d 2}<x l_{d}-\eta y \Leftrightarrow d_{c, d 2}>\left(x l_{d}-\eta y\right)^{-1 / \alpha}
\end{aligned}
$$

Equations (23) and (26) declare the amount of the interference that can be handled by the FD devices. Thus, they form the requirements to have an efficient FD-D2D link.

\section{APPENDIX B}

In the targeted region the denominator of both $R_{D 1}$ and $R_{D 2}$ are less than one. Thus, using the geometric series of $\left[\left(\frac{l_{c, d 1}}{l_{d} y}-1\right)^{-1}\right.$ and $\left.\left(\frac{l_{c, d 2}}{l_{d} x}-1\right)^{-1}\right]$ allows us to express the utility function defined in (15) as follows:

$$
\begin{aligned}
U(x, y)= & -\underbrace{\sum_{k=0}^{\infty}\left(\frac{l_{c, d 1}}{l_{d} y}\right)^{k} E_{1}\left(\frac{\eta x}{l_{c, d 1}}\right) e^{\frac{\eta x}{l_{c, d 1}}}}_{I_{1}}-\underbrace{\sum_{i=0}^{\infty}\left(\frac{l_{c, d 2}}{l_{d} x}\right)^{i} E_{1}\left(\frac{\eta y}{l_{c, d 2}}\right) e^{\frac{\eta y}{l_{c, d 2}}}}_{I_{2}} \\
& +\underbrace{\sum_{k=0}^{\infty}\left(\frac{l_{c, d 1}}{l_{d} y}\right)^{k} E_{1}\left(\frac{\eta x}{l_{d} y}\right) e^{\frac{\eta x}{l_{d} y}}}_{I_{3}}+\underbrace{\sum_{i=0}^{\infty}\left(\frac{l_{c, d 2}}{l_{d} x}\right)^{i} E_{1}\left(\frac{\eta y}{l_{d} x}\right) e^{\frac{\eta y}{l^{x}}}}_{I_{4}}
\end{aligned}
$$

From [14], the summation of convex functions is convex function. Hence, analyzing the convexity of $U(x, y)$ is equivalent to analyzing the convexity of $I_{1}, I_{2}, I_{3}$ and $I_{4}$.

The concavity of $I_{1}$ and $I_{2}$ can be established by studying the convexity of the function $I(x, y)=\sum_{k=0}^{\infty}\left(\frac{a}{y}\right)^{k} E_{1}(b x) e^{b x}$, where $a$ and $b$ are arbitrarily positive numbers. To that end, we derive the Hessian matrix of $I(x, y)$, denoted by $M_{I}$, as follows:

$$
\begin{aligned}
M_{I} & =\left[\begin{array}{ll}
I_{11} & I_{12} \\
I_{21} & I_{22}
\end{array}\right] \\
& =\left[\begin{array}{cc}
\frac{a^{k}\left(e^{b x} E_{1}(b x) b^{2} x^{2}-b x+1\right)}{y^{k} x^{2}} & \frac{k\left(e^{b x} E_{1}(b x) b x-1\right)}{y^{k+1} x} \\
\frac{k\left(e^{b x} E_{1}(b x) b x-1\right)}{y^{k+1} x} & \frac{k(k+1) a^{k} E_{1}(b x) e^{b x}}{y^{k+2}}
\end{array}\right]
\end{aligned}
$$

Based on the exponential integral properties given in [13, chap.5], we found that $I_{11}>0$ and $I_{11} I_{22}-I_{12} I_{21}>=0$. Now using the Sylvester's criterion [15] we can show that $I(x, y)$ is convex. Following the same procedure, the concavity of $I_{3}$ and $I_{4}$ can be established and thus $U(x, y)$ is concave function.

\section{ACKNOWLEDGEMENT}

The work done in this paper is supported by French National Research Agency (ANR) project ACCENT5 with grant agreement code: ANR-14-CE28-0026-02.

\section{REFERENCES}

[1] A. A. Al Haija and M. Vu, "Spectral efficiency and outage performance for hybrid d2d-infrastructure uplink cooperation," IEEE Transactions on Wireless Communications, vol. 14, no. 3, pp. 1183-1198, 2015.

2] Z. Zhang, K. Long, A. V. Vasilakos, and L. Hanzo, "Full-duplex wireless communications: challenges, solutions, and future research directions," Proceedings of the IEEE, vol. 104, no. 7, pp. 1369-1409, 2016.

[3] A. Sabharwal, P. Schniter, D. Guo, D. W. Bliss, S. Rangarajan, and R. Wichman, "In-band full-duplex wireless: Challenges and opportunities," IEEE Journal on Selected Areas in Communications, vol. 32, no. 9, pp. 1637-1652, 2014.

[4] K. E. Kolodziej, J. G. McMichael, and B. T. Perry, "Multitap rf canceller for in-band full-duplex wireless communications," IEEE Transactions on Wireless Communications, vol. 15, no. 6, pp. 4321-4334, 2016.

[5] L. Laughlin, C. Zhang, M. A. Beach, K. A. Morris, and J. Haine, "A widely tunable full duplex transceiver combining electrical balance isolation and active analog cancellation," in 2015 IEEE 81st Vehicular Technology Conference (VTC Spring), May 2015, pp. 1-5.

[6] V. Tapio, "System scenarios and technical requirements for full-duplex concept," DUPLO, Project Deliverable, 2013.

[7] K. T. Hemachandra, N. Rajatheva, and M. Latva-aho, "Sum-rate analysis for full-duplex underlay device-to-device networks," in 2014 IEEE Wireless Communications and Networking Conference (WCNC), April 2014, pp. 514-519.

[8] W. Cheng, X. Zhang, and H. Zhang, "Optimal power allocation for fullduplex d2d communications over wireless cellular networks," in 2014 IEEE Global Communications Conference, Dec 2014, pp. 4764-4769.

[9] B. Zuo, L. Jiang, C. He, and Z. Lian, "Power allocation optimization for full-duplex d2d communications underlaying cellular networks," in 2016 International Conference on Networking and Network Applications (NaNA). IEEE, 2016, pp. 103-108.

[10] S. Dang, G. Chen, and J. P. Coon, "Outage performance analysis of full-duplex relay-assisted device-to-device systems in uplink cellular networks," IEEE Transactions on Vehicular Technology, vol. 66, no. 5, pp. 4506-4510, 2017.

[11] X. Chai, T. Liu, C. Xing, H. Xiao, and Z. Zhang, "Throughput improvement in cellular networks via full-duplex based device-to-device communications," IEEE Access, vol. 4, pp. 7645-7657, 2016.

[12] V. Raghavan, S. V. Hanly, and V. V. Veeravalli, "Statistical beamforming on the grassmann manifold for the two-user broadcast channel," IEEE Transactions on Information Theory, vol. 59, no. 10, pp. 6464-6489, 2013.

[13] M. Abramowitz and I. A. Stegun, Handbook of Mathematical Functions with Formulas, Graphs and Mathematical Tables, 10th ed. Gaithersburg, MD, USA: National Bureau of Standards, 1972.

[14] S. Boyd and L. Vandenberghe, Convex optimization. Cambridge university press, 2004.

[15] R. A. Horn and C. R. Johnson, Matrix analysis. Cambridge university press, 2012. 